\title{
Papers
}

\section{Effectiveness of treatment for alcohol problems: findings of the randomised UK alcohol treatment trial (UKATT)}

\author{
UKATT Research Team
}

\begin{abstract}
Objective To compare the effectiveness of social behaviour and network therapy, a new treatment for alcohol problems, with that of the proved motivational enhancement therapy.

Design Pragmatic randomised trial.

Setting Seven treatment sites around Birmingham, Cardiff, and Leeds.

Participants 742 clients with alcohol problems; 689 (93.0\%) were interviewed at three months and $617(83.2 \%)$ at 12 months.

Interventions Social behaviour and network therapy and motivational enhancement therapy.

Main outcome measures Changes in alcohol consumption, alcohol dependence, and alcohol related problems over 12 months.

Results Both groups reported substantial reductions in alcohol consumption, dependence, and problems, and better mental health related quality of life over 12 months. Between groups we found only one significant difference in outcome, probably due to chance: the social network group showed significantly better physical health at three months. Non-significant differences at 12 months in the motivational group relative to the social network group included: the number of drinks consumed per drinking day had decreased by an extra 1.1 (95\% confidence interval - 1.0 to 3.2 ); scores on the Leeds dependence questionnaire had improved by an extra $0.6(-0.7$ to 2.0); scores on the alcohol problems questionnaire had improved by an extra 0.5 ( -0.4 to 1.4$)$; but the number of days abstinent from drinking had increased by $1.2 \%$ less $(-4.5 \%$ to $6.9 \%)$.

Conclusion The novel social behaviour and network therapy for alcohol problems did not differ significantly in effectiveness from the proved motivational enhancement therapy.
\end{abstract}

\section{Introduction}

Alcohol dependence and misuse are common and costly. In 2000 the estimated prevalence of alcohol dependence in the United Kingdom was 11.9\% among men and 2.9\% among women. ${ }^{1}$ The public costs of heavy drinking in England and Wales are about $£ 18000 \mathrm{~m}(\$ 32438 \mathrm{~m}$; $€ 26424 \mathrm{~m}){ }^{2}{ }^{2}$ Some 300 advice and counselling services, 100 day programmes, and nearly 200 residential programmes meet the resulting demand for treatment."

No British randomised trial of non-pharmacological treatments for alcohol problems has had the statistical power to detect even medium sized effects. ${ }^{4}$ To our knowledge only one randomised trial (the "matching alcoholism treatments to client heterogeneity" trial) of psychosocial treatments for alcohol problems has been able to detect small effects, by studying 1726 clients in nine treatment sites across the United States. ${ }^{5}$ Clients were randomly allocated to three manual based interventions, namely cognitive behavioural therapy, twelve step facilitation therapy, and motivational enhancement therapy. Cognitive behavioural therapy and twelve step facilitation therapy each comprised 12 sessions whereas the motivational enhancement therapy comprised four sessions, all over 12 weeks. Results showed substantial improvements in drinking outcomes for all three. Motivational enhancement therapy achieved outcomes essentially similar to those of the two more intensive treatments. ${ }^{56}$ The size of the trial meant that this was unlikely to have been a type 2 error.

This evidence and the increasing popularity of motivational enhancement therapy led to the proposal that this therapy should act as standard treatment in research on the effectiveness of treatment for alcohol problems. ${ }^{7}$ Meta-analyses have since confirmed the effectiveness of such treatments. ${ }^{8}$ These arguments led us to propose- and the Medical Research Council to accept - that motivational enhancement therapy should act as reference treatment within the United Kingdom alcohol treatment trial (UKATT). ${ }^{9}$ For ethical reasons we excluded a "no treatment" control group because we did not want to deprive participants of the known benefits of treatment.

To build on the US trial we exploited systematic reviews citing effective treatments that considered clients' social environment and support system. ${ }^{10}$ We designed and tested the social behaviour and network therapy specifically for our trial. ${ }^{11}$ The underlying theory is that overcoming alcohol problems needs support from family or friends. Thus we compared a novel social treatment with a strong theoretical and empirical basis with an established but briefer motivational treatment of proved effectiveness.

\section{Methods}

We carried out a pragmatic randomised trial in seven UK sites around Birmingham, Cardiff, and Leeds to test two main null hypotheses ${ }^{9}$ that socially based treatment (social behaviour and network therapy) and briefer, motivationally based treatment (motivational enhancement therapy) are equally effective and equally cost effective, notably in improving clients' quality of life (reported in the accompanying economic paper). ${ }^{12}$

The corresponding alternative hypotheses were both two sided. We were keen to derive recommendations for clinical

Full authorship details and collaborators are on bmj.com 
practice from the trial. With little research in this field we were open to recommend either therapy as the treatment of choice. If the therapies were similar in effectiveness and cost effectiveness, we were open to recommend that choice should depend on other factors, perhaps local.

\section{Interventions}

The sites nominated 76 candidates to train as therapists for the trial. To demonstrate suitability for training, the candidates submitted separate video recordings showing motivational skills and ability to work with clients' family and friends. We randomly assigned roughly twice as many therapists to the social behaviour and network therapy as to motivational enhancement therapy because social therapy takes about twice as much therapist time as motivational therapy. We trained 72 of the candidates in their allocated treatments through courses at Leeds lasting three days. To achieve accreditation, the trainees had to complete the supervised treatment of one or two clients and show competence through video recordings. The 52 therapists who achieved this continued to record treatment sessions on video and to receive supervision to encourage compliance with their allocated manual.

To cater for needs not addressed by the treatments, to clients awaiting randomisation we offered alcohol detoxification, treatment of organic disease, attention to emergency housing needs, and advice on financial matters. ${ }^{9}$

\section{Social behaviour and network therapy}

Social behaviour and network therapy comprises cognitive and behavioural strategies to help clients build social networks supportive of change. ${ }^{11}$ In our trial the therapy comprised eight 50 minute sessions over eight to 12 weeks.

\section{Motivational enhancement therapy}

In our trial, motivational enhancement therapy comprised three 50 minute sessions over eight to 12 weeks. ${ }^{9}$ It combined counselling in the motivational style ${ }^{13}$ with objective feedback. ${ }^{14}$ The therapy showed two main changes from the version used in the US study, ${ }^{14}$ both designed to keep it distinct from social behaviour and network therapy while retaining the features that led to the proposal that it act as standard treatment in research on treatment for alcohol problems. ${ }^{78}$ Firstly, we offered three sessions rather than four. Secondly, we allowed "significant others" to attend only the first session and to provide only confirmatory information.

\section{Inclusion and exclusion criteria}

We sought to include clients who would normally receive an offer of treatment from British treatment sites for alcohol problems. ${ }^{9}$ We excluded people aged under 16 ; people who were illiterate, could not name a contact, or intended to leave the area; people with uncontrolled psychotic illness or severe cognitive impairment; people for whom alcohol was not the main problem; and people who were receiving treatment for an alcohol problem.

\section{Screening, randomisation, and follow-up}

Clinical staff screened clients for eligibility. ${ }^{9}$ Potential participants were interviewed by the researchers to confirm eligibility and to gain written informed consent. The remote randomisation service at York used a computer "on line" to allocate consenting participants between therapy groups, stratified by site. Treatment was concealed until allocation. To make best use of available slots the service allocated clients between therapies
Table 1 Participant characteristics at randomisation. Values are numbers (percentages) unless stated otherwise

\begin{tabular}{|c|c|c|c|}
\hline Categorical characteristics & $\begin{array}{l}\text { Motivational } \\
\text { group }\end{array}$ & $\begin{array}{c}\text { Social } \\
\text { behaviour group }\end{array}$ & Total \\
\hline & $\mathrm{n}=422$ & $n=320$ & $n=742$ \\
\hline Mean (SD) age (years) & $41.8(10.1)$ & $41.4(10.0)$ & $41.6(10.1)$ \\
\hline Men & $315(74.6)$ & $235(73.4)$ & $550(74.1)$ \\
\hline White & $406(96.2)$ & $303(94.7)$ & 709 (95.6) \\
\hline \multicolumn{4}{|l|}{ Education: } \\
\hline Degree or equivalent & $45(10.7)$ & $29(9.1)$ & $74(10.0)$ \\
\hline No qualifications & $272(64.5)$ & $205(64.1)$ & $477(64.3)$ \\
\hline Marital status: & $\mathrm{n}=422$ & $\mathrm{n}=319$ & $\mathrm{n}=741$ \\
\hline Married, living with partner & $167(39.6)$ & $123(38.6)$ & $290(39.1)$ \\
\hline Married, not living with partner & $15(3.6)$ & $23(7.2)$ & $38(5.1)$ \\
\hline Single, in current relationship & $60(14.2)$ & $51(16.0)$ & $111(15.0)$ \\
\hline $\begin{array}{l}\text { Single, not in current } \\
\text { relationship }\end{array}$ & $180(42.7)$ & $122(38.2)$ & $302(40.8)$ \\
\hline Employment: & $\mathrm{n}=420$ & $\mathrm{n}=319$ & $\mathrm{n}=739$ \\
\hline Employed & $152(36.2)$ & $105(32.9)$ & $257(34.8)$ \\
\hline Sick or disabled & $146(34.8)$ & $106(33.2)$ & $252(34.1)$ \\
\hline Unemployed & 96 (22.9) & $86(27.0)$ & $182(24.6)$ \\
\hline Retired & $22(5.2)$ & $16(5.0)$ & $38(5.1)$ \\
\hline Non-employed & $4(1.0)$ & $6(1.8)$ & $10(1.4)$ \\
\hline Reported annual income: & $n=410$ & $n=314$ & $\mathrm{n}=724$ \\
\hline$>£ 20000$ & $73(17.8)$ & $64(20.4)$ & $137(19.0)$ \\
\hline$£ 15001-£ 20000$ & $45(11.0)$ & $20(6.4)$ & $65(9.0)$ \\
\hline$£ 10001-£ 15000$ & 57 (13.9) & $41(13.1)$ & $98(13.5)$ \\
\hline$£ 5001-£ 10000$ & 88 (21.5) & $63(20.1)$ & $151(20.9)$ \\
\hline$<£ 5000$ & $147(35.9)$ & $126(40.1)$ & $273(37.7)$ \\
\hline Goals reported at recruitment: & $n=422$ & $n=320$ & $\mathrm{n}=742$ \\
\hline To abstain & $226(53.6)$ & $177(55.3)$ & $403(54.3)$ \\
\hline To abstain through disulfiram* & $62(14.7)$ & $67(20.9)$ & $129(17.4)$ \\
\hline To use acamprosate $†$ & $85(20.1)$ & $58(18.1)$ & $143(19.3)$ \\
\hline Detoxification $\ddagger$ & $107(25.4)$ & $104(32.5)$ & $211(28.4)$ \\
\hline
\end{tabular}

${ }^{*}$ Creates expectation that drinking results in unpleasant reaction between alcohol and drug. †Suppresses craving for alcohol.

‡Done between screening and recruitment.

with probabilities proportional to the numbers of slots currently available for those treatments in the client's site. ${ }^{9}$

Researchers interviewed participants at home three and 12 months after entry to the trial, regardless of whether treatment was completed. ${ }^{9}$ Both schedules were similar to those completed before treatment. At 12 months we employed a new team of interviewers to ensure that they were blind to treatment allocation. We did not have the resources to do this at three months.

\section{Outcome measures}

Primary outcome measures were ${ }^{9}$ alcohol consumption, derived from form $90^{15}$ and summarised by number of drinks (that is, units of alcohol, equivalent to $8 \mathrm{~g}$ of ethanol) per drinking day and percentage of days abstinent $t^{5}$ alcohol dependence, measured by the Leeds dependence questionnaire ${ }^{16}$; alcohol related problems over the past three months, measured by the alcohol problems questionnaire ${ }^{17}$; and $\gamma$-glutamyl transferase, a liver function test measured by the Reflotron ${ }^{18}$-although this test is better used to monitor change after an abnormal score, it reputedly corroborates reported alcohol consumption.

Secondary outcome measures were associated with health related quality of life ${ }^{9}$ : the EQ-5D, a commonly used health status index ${ }^{19}$; the SF-36, a commonly used health profile ${ }^{20}$; and the general health questionnaire $28,{ }^{21}$ commonly used to measure psychological disturbance.

Sample size

We aimed to recruit 720 clients at seven sites. ${ }^{9}$ We sought to interview $80 \%$ of these after 12 months. Allowing for correlation 
Table 2 Mean (95\% confidence intervals) adjusted scores for primary and secondary outcomes across social behaviour group and motivational group combined at baseline and three and 12 months

\begin{tabular}{|c|c|c|c|}
\hline Outcome measures & Baseline $(n=742)$ & 3 months ( $n=689$ ) & 12 months ( $\mathrm{n}=617)$ \\
\hline Days abstinent (\%) & 29.5 (26.1 to 32.9$)$ & 42.7 (38.2 to 47.2$)$ & 46.0 (40.8 to 51.2$)$ \\
\hline No of drinks ${ }^{*}$ per drinking day & 26.8 (24.9 to 28.7$)$ & 17.9 (16.3 to 19.5$)$ & 19.2 (17.2 to 21.2$)$ \\
\hline Log $\gamma$-glutamyl transferase & 4.01 (3.86 to 4.16$)$ & 3.89 (3.77 to 4.01$)$ & 4.00 (3.83 to 4.17$)$ \\
\hline Leeds dependence questionnaire & 17.0 (15.9 to 18.1$)$ & 11.9 (10.8 to 13.0$)$ & 10.9 (9.6 to 12.2$)$ \\
\hline Alcohol problems questionnaire $†$ & 12.3 (11.7 to 12.9$)$ & 6.8 (6.1 to 7.5$)$ & 6.1 (5.3 to 7.0$)$ \\
\hline EuroQol (EQ-5D) & 0.574 (0.534 to 0.614$)$ & $0.629(0.592$ to 0.666$)$ & 0.607 (0.562 to 0.652$)$ \\
\hline General health questionnaire 28 & 40.0 (37.6 to 42.4) & 31.2 (28.7 to 33.7) & 29.9 (27.0 to 32.8) \\
\hline \multicolumn{4}{|l|}{ SF-36: } \\
\hline Mental component & 29.7 (28.0 to 31.4 ) & 36.6 (34.6 to 38.6 ) & 38.7 (36.5 to 40.9$)$ \\
\hline Physical component & 45.4 (44.0 to 46.8$)$ & 48.0 (46.8 to 49.2$)$ & 47.6 (46.2 to 49.0$)$ \\
\hline
\end{tabular}

Adjusted by analysis of covariance for centre, use of detoxification, and three goals reported at randomisation (to abstain, to abstain through disulfiram, and to use acamprosate); and corresponding baseline score for outcomes at 3 and 12 months.

*Units of alcohol, equivalent to $8 \mathrm{~g}$ of ethanol.

†Common items.

within sites and for the minor imbalance in the number of clients allocated to each therapy resulting from the corresponding imbalance in the numbers of therapists, our trial had at least $80 \%$ power using a $5 \%$ significance level to detect a standardised difference, either positive or negative, of less than one quarter (usually regarded as a small effect) between the therapies.

\section{Statistical analysis}

In accordance with our prespecified analysis plan we used analysis of covariance to take account of site (prestratification factor), use of detoxification, and drinking goal as reported at randomisation (poststratification factors) and to adjust for differences between groups in scores before randomisation. We analysed data by intention to treat. To assess whether attrition affected findings we undertook sensitivity analysis using the last recorded response from each respondent (last observation carried forward)

\section{Results}

Between 1999 and 2001 we recruited 742 participants with alcohol problems (figure) from clients of all ages above 16 and all social groups (table 1). Although only 211 (28.4\%) received detoxification between screening and recruitment (table 1), others had done so before screening, when levels of dependency were probably higher. We interviewed $689(93.0 \%)$ participants at three months and $617(83.2 \%)$ at 12 months (table 2). Of 125 participants lost to follow-up at 12 months, 12 had died, 35 did not respond, and 78 could not be traced or contacted.

Analysis of covariance showed that the therapy groups achieved similar outcomes at three and 12 months (table 3). As attrition was only $7 \%$ at three months and $17 \%$ at 12 months, sensitivity analysis using the last observation carried forward generated similar findings. The only significant difference we found was that after three months the adjusted mean physical component score of the SF-36 for clients in the social network group exceeded that of the clients in the motivational group by 1.31 (95\% confidence interval 0.05 to 2.57 ). As both mean scores were close to those at baseline (table 2), and because we invoked 18 distinct significance tests (table 3), it would be prudent to regard this as a possible random consequence of multiple comparisons.

To explore these findings further, we compared the mean adjusted scores at baseline and after three and 12 months for all who responded (table 2). At three months we found highly significant improvements in all patient outcomes except the EQ-5D and $\log \gamma$-glutamyl transferase. Baseline correlations between $\log \gamma$-glutamyl transferase and patient assessed outcomes were, however, all 0.3 or less.

The adjusted proportion of days on which clients reported that they had abstained improved from $29 \%$ to $43 \%$ at three months and to $46 \%$ at 12 months. Mean adjusted alcohol consumption reported by continuing drinkers fell from 27

Table 3 Mean (SE) adjusted scores for primary and secondary outcomes by allocated treatment at three and 12 months

\begin{tabular}{|c|c|c|c|c|c|c|}
\hline \multirow[b]{2}{*}{ Outcomes measures } & \multicolumn{3}{|c|}{3 months } & \multicolumn{3}{|c|}{12 months } \\
\hline & Motivational group & Social network group & $\begin{array}{l}\text { Difference in favour of } \\
\text { social group }(95 \% \mathrm{CI})\end{array}$ & Motivational group & Social network group & $\begin{array}{l}\text { Difference in favour of } \\
\text { social group }(95 \% \mathrm{Cl})\end{array}$ \\
\hline Days abstinent (\%) & $42.3(2.56)(n=393)$ & $43.2(2.63)(n=293)$ & 0.90 (-3.98 to 5.78) & $45.4(2.98)(n=351)$ & $46.6(3.06)(\mathrm{n}=261)$ & 1.19 (-4.50 to 6.88$)$ \\
\hline No of drinks per drinking day & $17.6(0.89)(\mathrm{n}=361)$ & $18.2(0.92)(\mathrm{n}=263)$ & $-0.53(-2.22$ to 1.17$)$ & $18.7(1.11)(n=303)$ & $19.8(1.15)(n=217)$ & $-1.14(-3.22$ to 0.95$)$ \\
\hline Log $\gamma$-glutamyl transferase & $3.87(0.070)(n=331)$ & $3.90(0.070)(n=259)$ & $-0.033(-0.163$ to 0.097$)$ & $4.01(0.0941)(n=293)$ & $4.00(0.0992)(n=214)$ & $0.010(-0.170$ to 0.189$)$ \\
\hline $\begin{array}{l}\text { Leeds dependence questionnaire } \\
(0-30,0=\text { best })\end{array}$ & $12.0(0.65)(n=367)$ & $11.8(0.67)(\mathrm{n}=275)$ & 0.24 (-0.99 to 1.46$)$ & $10.6(0.728)(n=332)$ & $11.2(0.766)(\mathrm{n}=231)$ & $-0.64(-2.02$ to 0.74$)$ \\
\hline $\begin{array}{l}\text { Alcohol problems questionnaire } \\
(0-23,0=\text { best })\end{array}$ & $6.85(0.41)(n=371)$ & $6.79(0.42)(n=280)$ & 0.06 (-0.71 to 0.83$)$ & $5.90(0.467)(n=326)$ & 6.38 (0.489) (n=233) & -0.48 ( -1.36 to 0.40$)$ \\
\hline EQ-5D (<0 to $1,1=$ best $)$ & $\begin{array}{c}0.631(0.021) \\
(\mathrm{n}=359)\end{array}$ & $\begin{array}{c}0.626(0.022) \\
(n=272)\end{array}$ & $-0.005(-0.045$ to 0.035$)$ & $0.623(0.025)(\mathrm{n}=317)$ & $0.592(0.026)(\mathrm{n}=230)$ & $-0.031 \quad(-0.079$ to 0.016$)$ \\
\hline $\begin{array}{l}\text { General health questionnaire } 28 \\
(0-84,0=\text { best })\end{array}$ & $31.6(1.43)(n=364)$ & $30.9(1.47)(n=274)$ & 0.71 (-1.98 to 3.40$)$ & $28.6(1.63)(n=324)$ & $31.2(1.72)(n=234)$ & $-2.64(-5.73$ to 0.44$)$ \\
\hline \multicolumn{7}{|l|}{$\begin{array}{l}\text { SF-36 (mean 50, SD 10, } \\
\text { higher=better): }\end{array}$} \\
\hline Mental component & $36.9(1.11)(n=341)$ & $36.3(1.12)(n=263)$ & $-0.56(-2.63$ to 1.50$)$ & $39.5(1.26)(n=299)$ & $37.9(1.30)(n=221)$ & $-1.56(-3.88$ to 0.76$)$ \\
\hline Physical component & $47.3(0.68)(\mathrm{n}=341)$ & $48.6(0.69)(\mathrm{n}=263)$ & 1.31 (0.05 to 2.57$)$ & $47.2(0.80)(\mathrm{n}=299)$ & $47.9(0.82)(n=221)$ & 0.68 (-0.79 to 2.16$)$ \\
\hline
\end{tabular}

Adjusted by analysis of covariance for corresponding baseline score, centre, use of detoxification, three goals reported at randomisation (to abstain, to abstain through disulfiram, and to use acamprosate. 
drinks per drinking day to 18 at three months and to 19 at 12 months. Reported mean adjusted scores on the Leeds dependence questionnaire fell from 17 to 12 at three months and to 11 at 12 months. Reported mean adjusted scores on the alcohol problem questionnaire fell from 12 to 7 at three months and to 6 at 12 months. Reported mental health also improved: the mean adjusted score of the mental component of the SF-36 rose from 30 to 37 at three months and to 39 at 12 months. We found no serious adverse events.

\section{Discussion}

Social behaviour and network therapy (a novel social treatment for alcohol problems) and motivational enhancement therapy (a motivational treatment of proved effectiveness), led to similar improvements in reported alcohol consumption, dependence, and problems, and in mental health, all of which were maintained over 12 months. Clients in both groups reported that total alcohol consumption had decreased by $48 \%$ at three months and by $45 \%$ at 12 months and that alcohol related problems had decreased by $44 \%$ at three months and by $50 \%$ at 12 months.

By recruiting more than 700 participants, our trial had power to detect small differences. Our trial was pragmatic in that it compared the effectiveness of treatments under routine conditions rather than their efficacy under controlled conditions. Internal validity was protected, notably by randomising therapists between therapies, randomising clients remotely, and keeping interviewers blind to treatment at 12 months. ${ }^{9}$ Since we treated motivational enhancement therapy as a reference treatment of proved effectiveness, we infer that social behaviour and network therapy is equally effective.

The intrinsic effects of the therapies may be less than those reported, however, for four reasons. Firstly, the scientific rigour

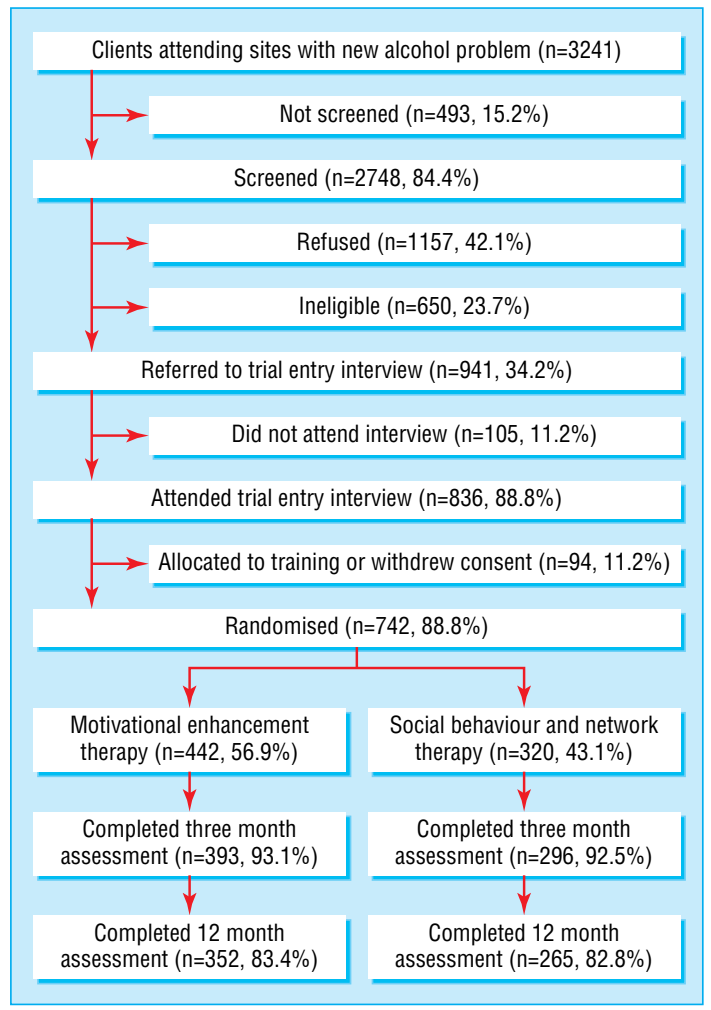

Flow of participants through trial

\section{What is already known on this topic}

Psychosocial treatment is generally effective for alcohol dependence and misuse

A multicentre trial and meta-analyses showed that motivational enhancement therapy, a brief psychosocial treatment, is effective

Systematic reviews have shown that some treatments involving members of the patient's social network are also effective

\section{What this study adds}

Social behaviour and network therapy did not differ in effectiveness from motivational enhancement therapy

Both therapy groups reported substantial reductions in drinking and associated problems and improved mental health

with which the trial design protected internal validity may have reduced external validity. Secondly, some improvement may have been due to regression to the mean. Thirdly, some improvement may have been the result of response bias. Finally, $\log \gamma$-glutamyl transferase, a test correlated with alcohol consumption, changed little over 12 months. However, this test adds little to careful history taking in clinical practice,,$^{22}$ and the US "matching alcoholism treatments to client heterogeneity" trial showed that it added little to self report in clinical trials. ${ }^{23}$

There are three other reasons for believing that the intrinsic effects of both therapies are real and sustained. Firstly, as there were three distinct steps between participants' attendance with a new alcohol problem and the baseline measurements that preceded randomisation (namely screening, referral, and interview; figure), bias due to regression to the mean is less likely to occur between baseline and subsequent measurements. Secondly, our participants consistently reported substantial reductions in alcohol consumption and consequent problems lasting at least 12 months. Finally, natural recovery from alcohol problems does occur but comparisons of treated and untreated samples imply that alcohol dependent individuals benefit from treatment..$^{24}$ Furthermore, evidence from trials shows that it is the intrinsic effect of motivational enhancement therapy rather than the non-specific effect of intervening that is responsible for its benefits. ${ }^{25}$

We can identify two main reasons why we found little difference between the therapies. Firstly, the evidence for the validity of self reports ${ }^{22}{ }^{23}$ and the evidence from outside our trial for the effectiveness of motivational enhancement therapy ${ }^{6-8} 2425$ strongly suggest that both therapies are effective. Secondly, both therapies add three common elements to the treatment process: a structured, published manual based on available scientific evidence; training of therapists at the Leeds Addiction Unit in a professional and rigorous atmosphere; and regular supervision of the therapists by separate teachers with considerable experience of treatment for alcohol problems. Hence therapists expected both therapies to yield good results.

In a separate economic paper we investigate the cost effectiveness of the therapies. ${ }^{12}$ Both social behaviour and network therapy and motivational enhancement therapy proved acceptable to clients, managers, and more than 50 therapists allocated between them at random. More importantly, our 
participants reported that the therapies were equally effective in reducing drinking and associated problems and in improving mental health.

We thank the participants for their contributions. The Medical Research Council established a trial steering committee to monitor the progress of the trial.

Contributors: AC was responsible for research and clinical management in the West Midlands. He contributed to the original grant application, the design, implementation, and management of the trial, analysing and interpreting data, and writing the paper. SC was responsible for designing and implementing the data management plan, and scanning, verifying, and validating data. He contributed to implementing the trial design, developing and implementing randomisation procedures, developing questionnaires and data collection instruments, analysing and interpreting data, and redrafting the paper. AF was responsible for designing and implementing the analysis plan, and validating data statistically. She contributed to implementing the trial design, developing and implementing randomisation procedures, developing data collection instruments, analysing and interpreting data, and redrafting the paper. CG was responsible for health economics. She contributed to the original grant application, the design, management, and implementation of the trial, analysing and interpreting data, and writing the paper. $\mathrm{NH}$ was responsible for research coordination. He contributed to the original grant application and participated in the design, implementation, and management of the trial, managing, analysing, and interpreting data, and writing the paper. RH was responsible for research and clinical management in South Wales. He contributed to the original grant application, the design, implementation and management of the trial, analysing and interpreting data, and writing the paper. VM contributed to validating, analysing, and interpreting data, and writing the paper. JO was responsible for trial coordination. He contributed to the original grant application, the design, implementation, and management of the trial, managing, analysing, and interpreting data, and writing the paper. SP was responsible for designing and implementing the health economic assessment. He contributed to implementing the trial design, developing procedures, questionnaires, and other instruments, analysing and interpreting data, and writing the paper. DR was responsible for research and clinical management in Leeds. He contributed to the original grant application, the design, implementation and management of the trial, analysing and interpreting data, and writing the paper. IR was responsible for trial methods. He contributed to the original grant application, the design, implementation, and management of the trial, managing, analysing, and interpreting data, and writing the paper. DR and IR act as guarantors. GS was responsible for designing and implementing trial management and quality assurance procedures, and coordinating the work of the treatment sites. He contributed to the implementation and management of the trial, developing procedures, questionnaires, and other instruments, designing and implementing the data management plan, verifying, validating, analysing, and interpreting data, and redrafting the paper. G Tober was responsible for therapist training and supervision. She contributed to the origina grant application, the design, implementation, and management of the trial, the management and analysis of video recordings of therapy within the trial, analysing and interpreting data, and writing the paper. The local research coordinators responsible for implementing and managing the trial were: $\mathrm{G}$ Thistlethwaite in the integrated treatment site in Leeds; TA across four treatment sites in South Wales; and CK across two treatment sites in the West Midlands. The main follow-up interviewers were: $\mathrm{LH}$ and AW in Leeds; RB and BJ in South Wales; and KF and MS in the West Midlands. RB and $\mathrm{BJ}$ later became local research coordinators in succession to TA, and MS in succession to CK. At the trial training centre in Leeds, OF and WM analysed the video recordings of therapy. These fieldworkers also contributed to developing procedures, questionnaires, and other instruments. Together with other authors they commented on successive drafts of the paper.
Funding: Medical Research Council, NHS Executive in England, Wales Office for Research and Development in Health and Social Care, and the Alcohol and Education Research Council.

Competing interests: None declared.

Ethical approval: All relevant local research ethics committees.

1 Singleton N, Bumpstead R, O'Brien M, Lee A, Meltzer H. Psychiatric morbidity among adults living in private households in 2000. London: Stationery Office, 2001.

2 Leontaridi R. Alcohol misuse: how much does it cost? London: Cabinet Office, 2003. www.strategy.gov.uk/files/pdf/econ.pdf (accessed 25 April 2005).

3 Alcohol Concern. Report on the mapping of alcohol services in England. London: Alcohol Concern, 2002.

4 Chick J, Ritson MB, Connaughton J, Stewart A, Chick J. Advice versus extended treatment for alcoholism: controlled study. Br J Addict 1988;83:159-70.

5 Project MATCH Research Group. Matching alcoholism treatments to client heterogeneity: project MATCH post-treatment drinking outcomes. J Stud Alcohol 1997;58:7-29

6 Project MATCH Research Group. Matching alcoholism treatments to client heterogeneity: project MATCH three-year drinking outcomes. Alcoh: Clin Exp Res 1998;22: 1300-11.

7 Finney JW. Limitations in using existing alcohol treatment trials to develop practice guidelines. Addiction 2000;95:1491-500.

8 Burke BL, Arkowitz H, Menchola M. The efficacy of motivational interviewing: a metaanalysis of controlled clinical trials. J Consult Clin Psychol 2003;71:843-61.

9 UKATT research team. United Kingdom alcohol treatment trial (UKATT): hypotheses, design and methods. Alcohol Alcohol 2001:36:11-21.

10 Miller WR, Andrews NR, Wilbourne P, Bennett ME. A wealth of alternatives: effective treatments for alcohol problems. In: Miller WR, Heather N, eds. Treating addictive behaviors. 2nd ed. New York: Plenum, 1998:203-16.

11 Copello A, Orford J, Hodgson R, Tober G, Barrett C on behalf of the UKATT research team. Social behaviour and network therapy: basic principles and early experiences. Addict Behav 2002;27:345-66.

12 UKATT Research Team. Cost effectiveness of treatment for alcohol problems: findings of the randomised UK alcohol treatment trial (UKATT). BMJ 2005;331:544-8.

13 Miller WR, Rollnick S. Motivational interviewing: preparing people for change. 2nd ed. New York: Guilford Press, 2002

14 Miller WR, Zweben A, DiClemente C, Rychtarik R. Motivational enhancement therapy: clinical research guide for therapists treating individuals with alcohol abuse and dependence. Project MATCH monograph 2. DHHS Publication No (ADM) 92-1894. Washington, DC: Department of Health and Human Services, 1992.

15 Miller WR. Form 90: a structured assessment interview for drinking and related behaviors. Project MATCH monograph 5. Rockville, MD: National Institute on Alcohol Abuse and Alcoholism, 1996.

16 Raistrick D, Bradshaw J, Tober G, Weiner J, Allison J, Healey C. Development of the Leeds dependence questionnaire (LDQ) to measure alcohol and opiate dependence in the context of a treatment evaluation package. Addiction 1994;89:563-72.

17 Drummond DC. The relationship between alcohol dependence and alcohol related problems in a clinical population. Br J Addict 1990;85:357-66.

18 Boehringer Mannheim Diagnostics. Reflotron client management system: accuracy and precision data. Indianapolis: Boehringer Mannheim, 1989

19 Brooks R. EuroQol: the current state of play. Health Policy 1996;37:53-72.

20 Ware JE, Sherbourne CD. The MOS 36-item short-form health survey (SF-36) I-conceptual framework and item selection. Med Care 1992;30:473-83.

21 Goldberg DP. The detection of psychiatric illness by questionnaire. Oxford: Oxford University Press, 1972.

22 Hillman A, Sykes RAD, McConnell AA. Limitations in the use of gamma-glutamyl transferase estimations in alcohol dependent subjects. Alcohol Alcohol 1998.33.626-30.

23 Babor T, Steinberg K, Del Boca F, Anton R. Talk is cheap: measuring drinking outcomes in clinical trials. J Stud Alcohol 2000;61:55-63.

24 Weisner C, Matzger H, Kaskutas LA. How important is treatment? One-year outcomes of treated and untreated alcohol-dependent individuals. Addiction 2003;98:901-11.

25 Sellman JD, Sullivan PF, Dore GM, Adamson SJ, MacEwan I. A randomised controlled trial of motivational enhancement therapy (MET) for mild to moderate alcohol dependence. J Stud Alcohol 2001;62:389-96.

(Accepted 27 June 2005)

bmj.com 2005;331:541

See web extra for full authorship details

Correspondence to: Jim Orford, professor of clinical and community psychology, School of Psychology, University of Birmingham, Birmingham B15 2TT j.f.orford@bham.ac.uk 